\title{
HCE: A New Channel Exchange Scheme for Handovers in Mobile Cellular Systems
}

\author{
DINESH K. ANVEKAR* ${ }^{*}$ and S. SANDEEP PRADHAN \\ Department of Electrical Communication Engineering \\ Indian Institute of Science, Bangalore 560012, India. \\ (Email:dka@ece.iisc.ernet.in)
}

\begin{abstract}
A new scheme for increasing handover success rate in mobile cellular systems is presented. The scheme involves exchange of channels between two mobiles which are moving in opposite directions across the handover area of adjacent cells. Performance evaluation of the new scheme in comparison with the conventional no-channel-exchange scheme is carried out by computer simulation of call traffic in two different two-cell models.
\end{abstract}

\section{Introduction}

During the last decade, several handover policies and channel assignment strategies have been proposed to minimize handover failures in mobile cellular communication systems [1]-[6]. At the present state of the art, all handover requests to a base station from mobiles of the adjacent cells are enqueued in a common queue, and processed based on some prioritization criteria and the availability of free channels. The only possibility for a channel to be made available to waiting handover requests in a cell is when a channel is released after natural termination of a call or after a handover request from a mobile of the current cell either succeeds or fails. However, it is possible for a mobile which has requested for handover to an adjacent cell to exchange its channel with that of another mobile, if any, moving from that cell to the current cell. Thus, both the mobiles will be successfully handed over without any channel being explicitly released. This is the basis of the new channel exchange scheme presented in this paper. The minimization of handover failure probability achievable by the new handover channel exchange (HCE) scheme is demonstrated here by computer simu-

\footnotetext{
${ }^{*}$ Presentation of this work by the author in the conference was made possible by a grant from Silicon Automation Systems (India) Pvt. Ltd, 3008, 12th "B" Main, 8th Cross, HAL 2nd stage, Indiranagar, Bangalore-560 008, India.
}

lation of call traffic with two different two-cell models.

\section{The HCE Scheme}

Consider the handover region between these two adjacent cells is shown schematically in Fig. 1. Here, the two cells are physically separated by the boundary line b12. The region between the two lines b1 and b2 represents the handover area. Beyond the right of line $b 1$, the radio power received by a mobile from base station $\mathrm{BS}_{1}$ is not sufficient to guarantee error-free communication. Similarly, the line $\mathrm{b} 2$ marks the point upto which the radio power received by a mobile from the base station $\mathrm{BS}_{2}$ is just sufficient for reliable communication. In this paper, a mobile is denoted by $\mathrm{M}_{i}(\mathrm{j}, \mathrm{k})$ which means the $\mathrm{i}$-th mobile holding a channel belonging to base station $\mathrm{BS}_{j}$ and moving towards. the base station $\mathrm{BS}_{k}$.

Suppose at the point of time corresponding to Fig. 1, both base stations $\mathrm{BS}_{1}$ and $\mathrm{BS}_{2}$ have no free channels available. Then, if the situation continues, after a certain period of time, the mobile $M_{1}(2,1)$ will cross $b 2$ and result in a handover failure, and its channel is released in cell 2. The base station $\mathrm{BS}_{2}$ can then assign this channel to the mobile $\mathrm{M}_{1}(1,2)$ if the latter is still in the handover area. The mobiles are served in this manner in a conventional channel allocation scheme. In the HCE scheme, however, the mobiles are allowed to exchange their channels if they are moving in opposite directions in the handover area. Thus, with the situation shown in Fig. 1, the channels held by the mobiles $M_{1}(1,2)$ and $M_{1}(2,1)$ are exchanged. This results in handover success for both the mobiles instead of for one mobile as in the conventional scheme.

In the HCE scheme, there is a close interaction between neighbouring base stations. A base station $\mathrm{BS}_{i}$ enqueues a handover request from a mobile moving from cell $j$ to cell $i$ in a separate queue $Q_{i j}$. Similarly, the base station $\mathrm{BS}_{j}$ maintains a queue $\mathrm{Q}_{j i}$ for handover requests 


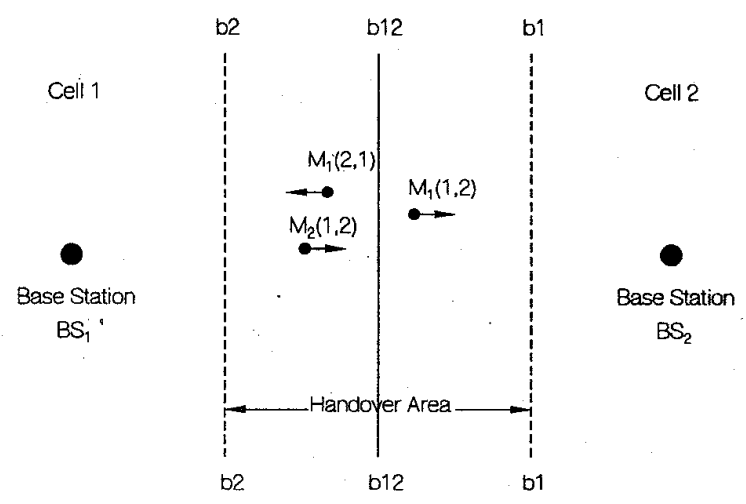

Figure 1: Schematic representation of handover area between two cells

from mobiles moving from cell $\mathrm{i}$ to cell $\mathrm{j}$. The two base stations $\mathrm{BS}_{i}$ and $\mathrm{BS}_{j}$ together process the queues $\mathrm{Q}_{i j}$ and $Q_{j i}$ so as to result in exchange of channels for suitably prioritized entries in the queues. The prioritization of the enqueued handover requests can follow any of the reported schemes,e.g., the measurement-based prioritization scheme of [6]. It can be easily seen that because of the channel exchange scheme, at no time can both $Q_{i j}$ and $\mathrm{Q}_{j i}$ be non-empty. A handover request from a mobile in cell $i$ to the base station of cell $j$ is processed in any of the following three ways:

(i) If a free channel is available in cell $\mathbf{j}$, it is assigned to the mobile; this results in handover success

(ii) If a free channel is not available in cell $j$ and $Q_{i j}$ is empty, then the handover request is enqueued in $Q_{j i}$

(iii) If a free channel is not available in cell $j$ and $Q_{i j}$ is non-empty, then the mobile is made to exchange its channel with the channel held by the mobile whose handover request has the highest priority in $Q_{i j}$

Once enqueued, a handover request can lead to handover success if a channel is released in the corresponding cell or if a channel is exchanged with a mobile of that cell. However, if a channel is not made available to a handover request within the interval during which it traverses the handover area, it results in a handover failure. The enqueued handover requests are periodically prioritized based on radio power measurements received from the mobiles. At any time if a free channel is available it is assigned to the highest priority mobile. Also, if possible, a channel exchange is initiated for the highest priority mobile in the queue. Timed-out handover requests which corresond to handover failures are periodically deleted from the queue. New call requests are just blocked if free channels are not available. However, a free channel is assigned to a new call request only if the handover request queue is empty. Thus, in the HCE scheme, as in the other handover prioritization schemes [3]-[6], a handover request has a higher priority than new call requests.

\section{Simulation Models}

In the simulation models, the call traffic in two adjacent cells of a mobile cellular system are considered as this is sufficient to indicate the comparative benefit of using handover channel exchanges. In each cell, the new calls are assumed to arrive at Poisson rate with a mean of $\lambda$, i.e., the inter-arrival time between two calls is assumed to be exponentially distributed with a mean of $1 / \lambda$. The mean inter-arrival time is varied to study the effect of increased offered traffic on the probabilities of call blocking and handover failure. When a channel is assigned to a new call or a handover call, it is assumed that the channel is held by the call for a duration $t_{c}$ which is exponentially distributed with a mean of $1 / \mu$. The two different simulation models considered in this work differ in the way the handover call traffic is generated. In the first model (SM1), a fraction (f) of new calls is assumed to result in handover requests to the other cell, after the elapse of time $t_{c}$. A handover call holds a channel for the duration $t_{c}$ and then terminates the call. In the second simulation model (SM2), whether a new call or a handover call results in handover request to the other cell is dependent on another time period $t_{h}$, which is assumed to be exponentially distributed with a mean of $1 / \sigma$. The period $t_{h}$ corresponds to the duration a mobile spends in a call before requesting for a handover to the other cell. Thus, if $t_{c} \leq t_{h}$, the call is terminated within the current cell; otherwise, a handover request is generated after holding the channel for a duration $t_{h}$ in the current cell. With this model, a call may result in several handovers between the two cells before it is either naturally terminated or forced to terminate due to handover failure.

The model for the motion of mobiles and the variation of radio power levels within the handover area is assumed to be the same as used in [6]. With this model, a mobile with the highest ratio of radio power levels received from the target base station and the current base station has the highest priority for either an allocation of a free channel or for an exchange of channel with another mobile. Also, the mobiles are assumed to move with uniform 
Table 1: Parameters and assumptions of the simulation models

\begin{tabular}{|c|c|}
\hline No. of channels per cell & 50 \\
\hline New call generation & $\begin{array}{l}\text { Poisson distributed ar- } \\
\text { rival rate }\end{array}$ \\
\hline $\begin{array}{l}\text { Channel holding time } \\
\left(t_{c}\right)\end{array}$ & $\begin{array}{l}\text { Exponentially } \\
\text { distributed with a mean } \\
\text { of } 1 / \mu=100 \text { sec. }\end{array}$ \\
\hline $\begin{array}{l}\text { Handover request gener- } \\
\text { ation time }\left(t_{h}\right)\end{array}$ & $\begin{array}{l}\text { Exponentially } \\
\text { distributed with a mean } \\
\text { of } 1 / \sigma=60 \mathrm{sec} \text {. }\end{array}$ \\
\hline $\begin{array}{l}\text { Channel assignment } \\
\text { strategy for cells }\end{array}$ & Fixed \\
\hline $\begin{array}{l}\text { Radio power degradation } \\
\text { within the handover area }\end{array}$ & Monotonic \\
\hline $\begin{array}{l}\text { Duration of handover } \\
\text { area traversal }\end{array}$ & $\begin{array}{l}\text { Normally dis- } \\
\text { tributed with a mean of } \\
10 \text { sec. and a standard } \\
\text { deviation of } 2 \mathrm{sec} \text {. }\end{array}$ \\
\hline
\end{tabular}

speed within the handover area.

The major parameters and assumptions characterizing the simulation models are given in Table 1 . The above models have been simulated using the Simscript II language. The results of simulation are presented in the next Section.

\section{Simulation Results}

The simulation was run with different new call arrival rates. The number of new call requests simulated in each cell was one million. The statistical results were calculated as averages for both the cells. Figs 2-5 show the simulation results for the model SM1. It is clear from these Figures that the HCE scheme results in lower handover failure probability without greatly affecting call blocking rate. The simulation results for the model SM2 are shown in Figs 6-9. The improvement in handover success rate due to channel exchanges is clear from Fig. 6. As seen here, the reduction in handover failure probability increases with the increase in the new call arrival rate. At a call traffic of 45 calls/minute, the new scheme reduces handover failure probability to almost half of that without channel exchanges.

As clear from Fig. 7, the HCE scheme results in lower call blocking probability as compared to the scheme without channel exchanges. Also, the reduction in call blocking probability increases with the increase in new call arrival rate. These reductions in call blocking probability are possible because in the new scheme a part of handover traffic is managed with channel exchanges and as a consequence, the probability of assignment of released channels to new calls increases. The higher reduction in call blocking probability at higher new call arrival rates is due to increase in number of handover channel exchanges at those new call arrival rates.

A plot of call dropping probability versus new call arrival rate is shown in Fig. 8. Call dropping is defined here as failure of a call in progress due to handover failure. As a direct result of reduction in handover failure probability, the HCE scheme has significantly lower call dropping probability as compared the scheme without channel exchanges.

The role of handover channel exchanges in increasing the number of successful calls is clear from Fig. 9. For example, at a new call arrival rate of 45 calls/minute, nearly $15 \%$ of calls are successful because of handover exchanges.

The superiority of the HCE scheme is clearly evident from the simulation results discussed above.

\section{Conclusion}

The HCE scheme presented in this paper improves handover success rate by exchanging channels between two mobiles moving in opposite directions across the handover area of adjacent cells. The improvement in cellular system performance by the use of the new scheme has been demonstrated by simulation of call traffic in two adjacent cells of a mobile cellular system. The simulation results show that the new scheme greatly minimizes handover failure probability, with no detrimental effect on call blocking probability. These results clearly show the utility of the HCE scheme in modern mobile cellular systems.

\section{References}

[1] S.Tekinay and B.Jabbari; "Handover policies and channel assignment strategies in mobile cellular networks", IEEE Commun. Mag., vol.29, no.11, Nov.1991.

[2] D.K.Anvekar,P.Agrawal, and B.Narendran, "A Traffic-driven Channel Reservation Scheme for Handovers in Mobile Cellular Networks", Proceedings of 
the Sixth International Conference on Wireless Communications, July 1994, Calgary, Alberta, Canada.

[3] D.K.Anvekar and P.Agrawal, "A New Criterion for Processing Handover Requests in Mobile Cellular Networks", Proceedings of the IEEE International Conference on Personal Wireless Communications, Aug.1994, India.

[4] P.Agrawal, D.K.Anvekar, and B.Narendran, "Minimizing Cellular Handover Failures without Channel Utilization Loss", Proceedings of Globecom'94, San Fransisco, USA.

[5] P.Agrawal, D.K.Anvekar, and B.Narendran, "Optimal Prioritization of Handovers in Mobile Cellular Networks", Proceedings of the Fifth IEEE International Symposium on Personal, Indoor and Mobile Radio Communications, Sept. 1994, The Hague, The Netherlands.

[6] S.Tekinay and B.Jabbari, "A measurement-based prioritization scheme for handovers in mobile cellular networks", IEEE J. Select. Areas Commun., vol.10, no.8, Oct.1992.

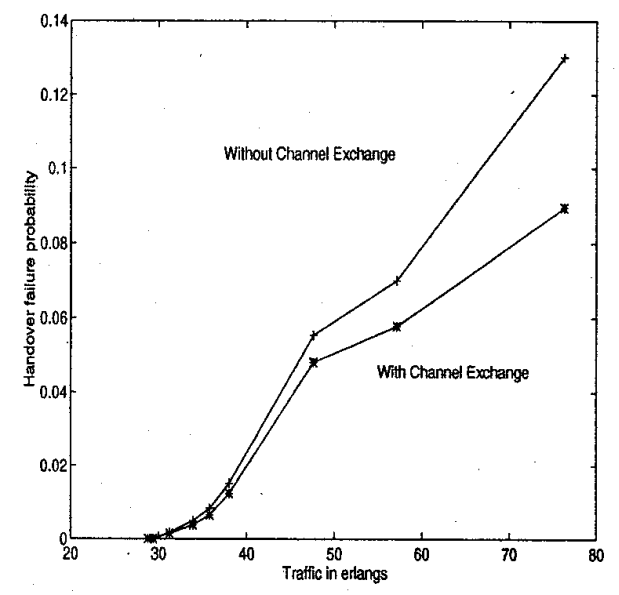

Figure 2: Handover failure probability for model SM1 with $\mathrm{f}=0.25$

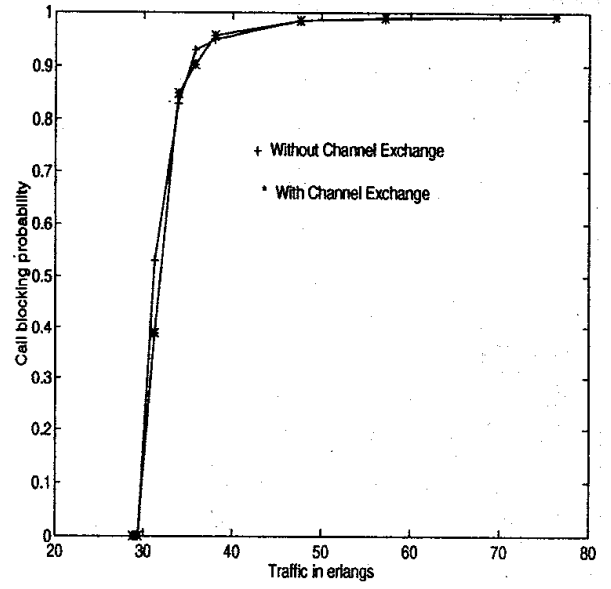

Figure 3: Call blocking probability for model SM1 with $\mathrm{f}=0.25$.

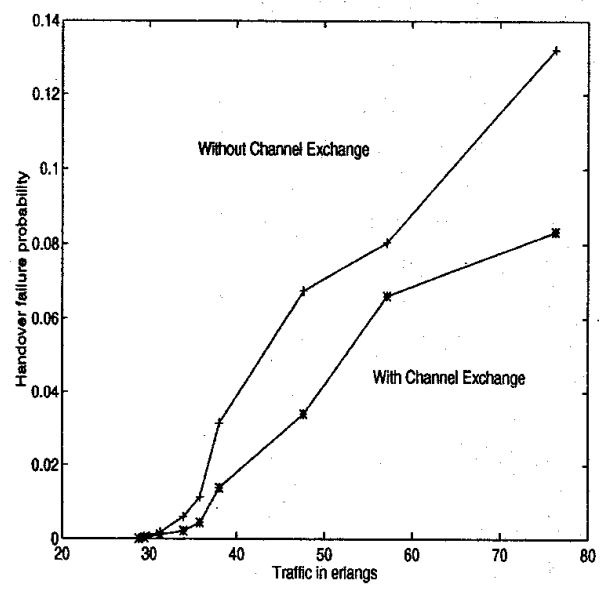

Figure 4: Handover failure probability for model SM1 with $\mathrm{f}=0.5$ 


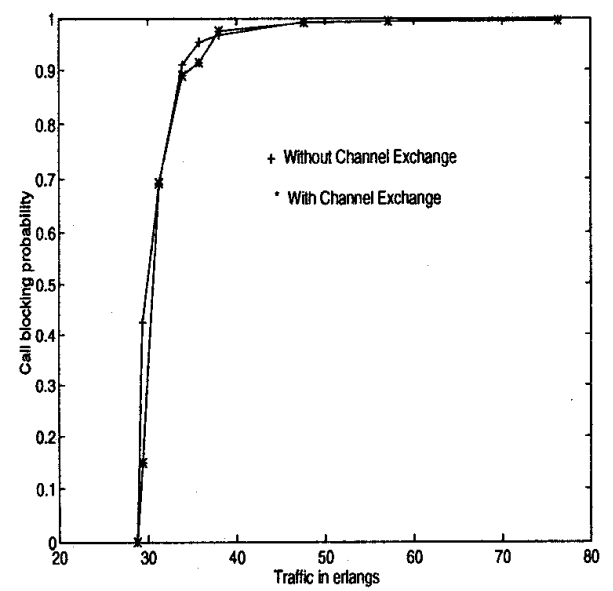

Figure 5: Call blocking probability for model SM1 with $\mathrm{f}=0.5$

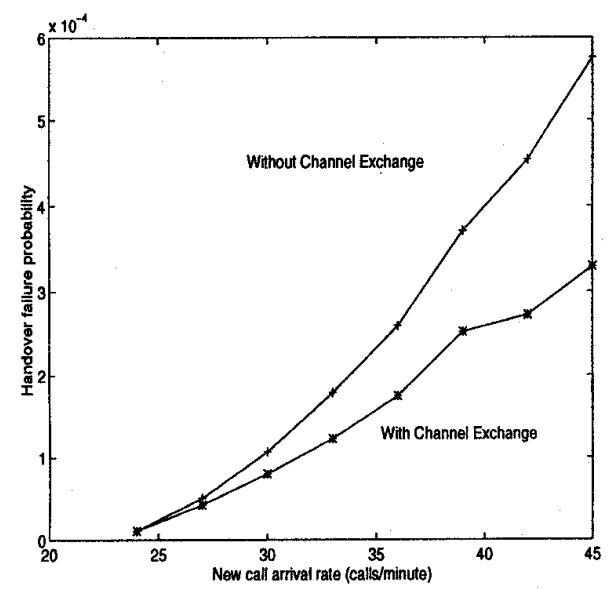

Figure 6: Handover failure probability for model SM2

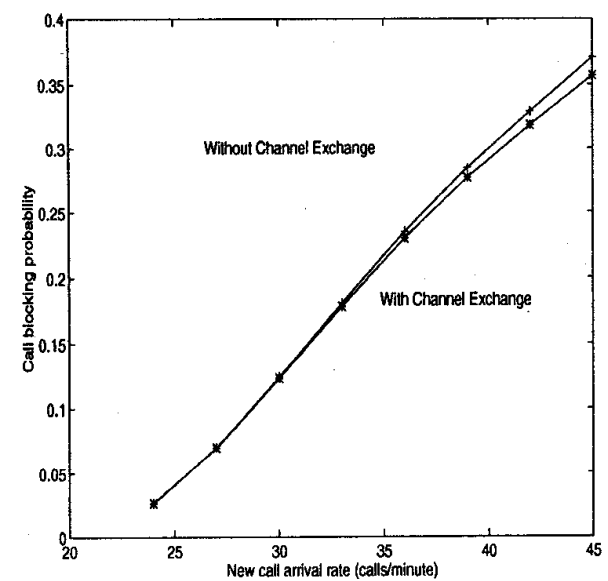

Figure 7: Call blocking probability for model SM2

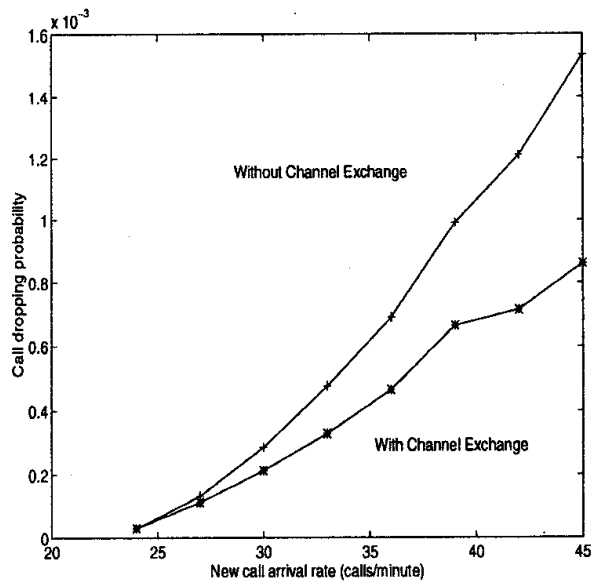

Figure 8: Call dropping probability for model SM2

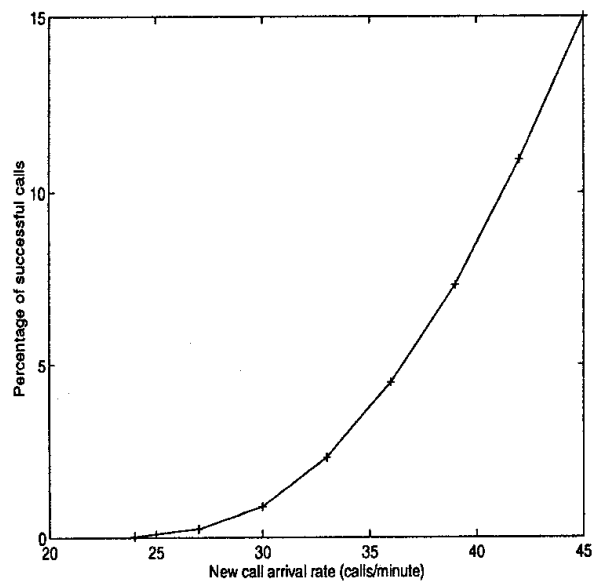

Figure 9: Percentage of calls in model SM2 successful due to handover channel exchange 